\title{
Evaluation of the Results of Patients Who Were Treated with Laparoscopic Simple Tunnel Hysterosacropexy Method to Prevent Uterine Prolapse: A New Approach
}

\author{
Erdal SEKER', Evindar ELCI²
}

Ankara, Turkey

\section{ABSTRACT}

OBJECTIVES: To evaluate the results of patients with uterine prolapse who underwent laparoscopic simple tunnel hysterosacropexy as uterine protective surgery. In this surgical procedure, the parietal peritoneum which was cut off in traditional surgery is preserved.

STUDY DESIGN: Data from women with apical prolapse of stage 2 or higher who underwent laparoscopic simple tunnel hysterosacropexy between October 1, 2017, and March 31, 2019, at the University of Health Sciences Diyarbakır Gazi Yasargil Education and Research Hospital, Diyarbakır, Turkey was retrospectively assessed. Patients were treated with laparoscopic simple tunnel hysterosacropexy; the meshes were anchored to the posterior cervical area, even in the presence of advanced multi-compartment vaginal prolapse. Data on the prolapse stage and urogenital functions were collected through clinical examinations, questionnaires at baseline, and 6 months after the operation. Results were analyzed using Wilcoxon Signed Ranks Test and Based on positive ranks. Data are presented as mean, minimum, maximum or percentage according to variables.

RESULT: Overall, 12 women were included in the analysis; the mean follow-up was 6 months. There were significant improvements in the complaints and POP-Q values of patients included in the study. There were not any complications during the intraoperative period and postoperative follow-ups. During the first six-month follow-up, there were not any recurrence of prolapse. The mean operative time was 146 minutes; there were no intraoperative visceral or vascular injuries. There was no recurrence or vaginal erosion.

CONCLUSION: In patients with stage 2 and more severe uterine prolapse, laparoscopic simple tunnel hysterosacropexy can be performed without cutting the peritoneum. Since the peritoneum is not cut during the surgery; intestinal injury prevalence and mesh exposure rates are lower.

Keywords: Hysterosacropexy, Laparoscopy, Prolapses

Gynecol Obstet Reprod Med 2021;27(2):163-168

\section{Introduction}

Uterovaginal prolapse has multifactorial etiology, but appears to be related to defects in the integrity of the uterosacralcardinal ligament complex and has traditionally been repaired by vaginal hysterectomy $(1,2)$. However, clinical experience

\footnotetext{
${ }^{1}$ Department of Obstetrics and Gynecology, Ankara University, Ankara, Turkey.

2 Department of Obstetrics and Gynecology, Siirt State Hospital, Siirt, Turkey Address of Correspondence: Erdal Seker

Department of Obstetrics and Gynecology, Ankara University, 06590 Ankara, Turkey. erdalseker84@gmail.com
}

Submitted for Publication: 27.04.2020 Revised for Publication:29.07.2020 Accepted for Publication: 26.10.2020 Online Published: 02.08.2021 ORCID IDs of the authors: $\quad$ ES: 0000-0001-9818-0414 EE: 0000-0001-5791-715X

\begin{tabular}{cc}
\hline Quick Response Code: & Access this article online \\
\cline { 2 - 2 } & Website: www.gorm.com.tr \\
e- mail: info@gorm.com.tr \\
\hline
\end{tabular}

showed that vaginal hysterectomy alone might fail to correct POP, as approximately $40 \%$ of patients presented other defects and $30 \%$ required repeated surgery. Therefore, hysterectomy is currently often combined with a vault suspending procedure (3-6).

Laparoscopic hysterosacropexy has become the standard treatment for symptomatic uterovaginal prolapse. In comparison with laparotomy, the laparoscopic hysterosacropexy has similar re-operation rates and similar recurrence rates, lower surgical morbidity, but otherwise similar anatomical and functional results (7). Previous studies comparing laparoscopic sacrocolpopexy (LSC) to abdominal sacrocolpopexy (ASC) showed less blood loss and significantly shorter hospital stay in the laparoscopic group. Simple laparoscopic hysterosacropexy has proven to be effective and beneficial (8).

How to cite this article: Seker E. and Evindar E. Evaluation of the Results of Patients Who Were Treated with Laparoscopic Simple Tunnel Hysterosacropexy Method to Prevent Uterine Prolapse: A New Approach. Gynecol Obstet Reprod Med 2021;27(2):163-168 
Macroporous, non-absorbable, and monofilament prolene mesh is generally used for fixation. Promontofixation also has two methods: the first one is to fix with tacker; the second is to fix with prolene suture. Closure of the peritoneum is preferred. Subcutaneous mesh application is a new method and can be performed without cutting the peritoneum. Peritoneal integrity is not impaired with this method. This not only reduces the operation time, but it also decreases mesh erosion.

The main difference between the laparoscopic simple tunnel hysterosacropexy (LSTHS) and the traditional surgery is to develop a space under the parietal peritoneum from promontory to the uterine cervix.

We retrospectively analyzed the life quality of the patients who underwent LSTHS.

\section{Material and Method}

Our study presents a retrospective descriptive study of data from women who underwent LSTHS at the Health Sciences University Diyarbakır Gazi Yasargil Education and Research Hospital, Diyarbakır, Turkey, between October 1, 2017, and March 31, 2019. Patients were informed on the new operation method and written consent was obtained for surgery, video recording, and personal data collection.

Women who met the following criteria were included in the study: Pelvic Organ Prolapse Apical vaginal prolapse of stage 2 or higher as determined by the Quantitative (POP-Q) mediator; treatment with LSTHS in accordance with the protocol of the urogynecology unit; and no prior sacropexy history. Patients who have had prior surgery regarding prolapses were not included in the study. Also, patients who have urinary incontinence complaints, and patients who have undergone incontinence surgery were not included in the study.

Results were analyzed using Wilcoxon Signed Ranks Test and Based on positive ranks. Data are presented as mean, minimum, maximum, or percentage according to variables.

Patients were admitted the day before the operation for bowel preparation if necessary. Anesthesia was standardized in favor of general anesthesia, if possible.

All operations were performed by a single experienced gynecologist.

We obtained consent from the ethics committee of our hospital.

We did not administer antibiotics before or after surgery.

The patient was placed under general endotracheal anesthesia in the lithotomy position. After routine preparation, a sterile catheter was placed in the bladder, and bullet forceps were placed upon the cervix to manipulate the uterus during the procedure. A $10 \mathrm{~mm}$ trocar was inserted directly into an incision in the umbilical crease without the use of a Veress needle and carbon dioxide was insufflated into the peritoneum.
After insertion of the $90^{\circ}$ camera, three more trocars were placed into the lower abdomen (two $5 \mathrm{~mm}$ trocars were placed on the left side of the patient and one $5 \mathrm{~mm}$ trocar on the right side of the patient). Pelvic and abdominal organs were inspected. The patient was placed in the Trendelenburg position to ensure the visibility of the whole pelvis.

The promontory was exposed. After the presentation of the right ureter, the peritoneum was opened over the sacrum on the promontory level and the anterior longitudinal ligament of the spine was exposed without injuring the inferior hypogastric plexus of the median sacral artery (Figure 1). Then, an incision was made on the parietal peritoneum of the dorsal wall of the cervix uteri and it was partially separated from the cervix (Figure 2). A retroperitoneal tunnel was created from the right side of the promontory. While creating this tunnel, the peritoneum was not cut. The tunnel was advanced to where the right sacrouterine ligament was attached to the cervix. The right ureteral peristalsis was checked and it was made sure it was not damaged. First, yellow color fatty tissue under the peritoneum was seen. Afterward, fatty tissue is dissected bluntly with the LigaSure ${ }^{\mathrm{TM}}$ (Valleylab Inc., Boulder, CO, USA) tool. These dissections were advanced to the posterior cervix. Peritoneum and sub-peritoneal ureter peristalsis was checked. (Figure 3). A retroperitoneal tunnel was formed between the promontory and the cervix uteri, following the anatomical location of the right uterosacral ligament. Later, the laparoscopic forceps tool was advanced from the tunnel to the posterior cervix. A macroporous, monofilament and nonabsorbable polypropylene mesh were used for the procedure. The proximal end of the graft was pulled through the retroperitoneal tunnel by the laparoscopic needle holder instrument (Porte Aiguilles) (Figure 4). Then the distal end of the web was attached to the cervix with prolene suture 2-0. The proximal end of the graft was attached to the right side of the longitudinal anterior ligament of the sacral spine with prolene suture 2-0 (Figure 5). All excessive mesh was removed. The peritoneum over the sutured mesh was closed with absorbable Vicryl ${ }^{\circledR} 2-0$ sutures (Figure 6 and 7). A $5 \mathrm{~mm}$ LigaSure $^{\mathrm{TM}}$ instrument was used during the procedure.

Hemostasis was ensured, all instruments were removed from the abdomen under visual control and the incisions were closed in a standard fashion (Figure8).

Then, the patients underwent urogynecologic examination. Colporrhaphy anterior, colporrhaphy posterior, and perineoplasty were performed in patients with vaginal width, cystocele or rectocele.

Foley catheter was removed on the $6^{\text {th }}$ hour of the postoperative period. The patients were followed-up for spontaneous diuresis. Postoperative abdominal USG and renal USG were routinely performed. The patients were evaluated for renal pelvic ectasia and hematoma. We have obtained the permission of the ethics committee of Gazi Yasargil Education and Research Hospital, 368, on 14.10.2019. 


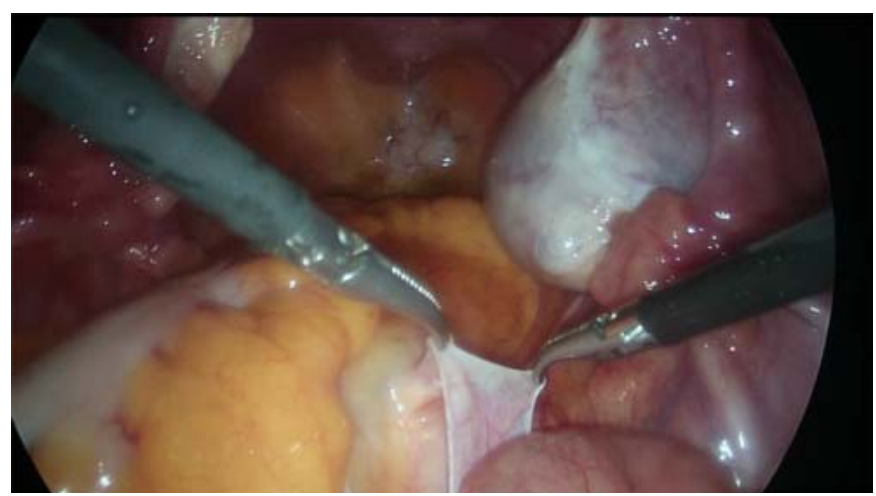

Figure 1: Preparation of the sacropromontory

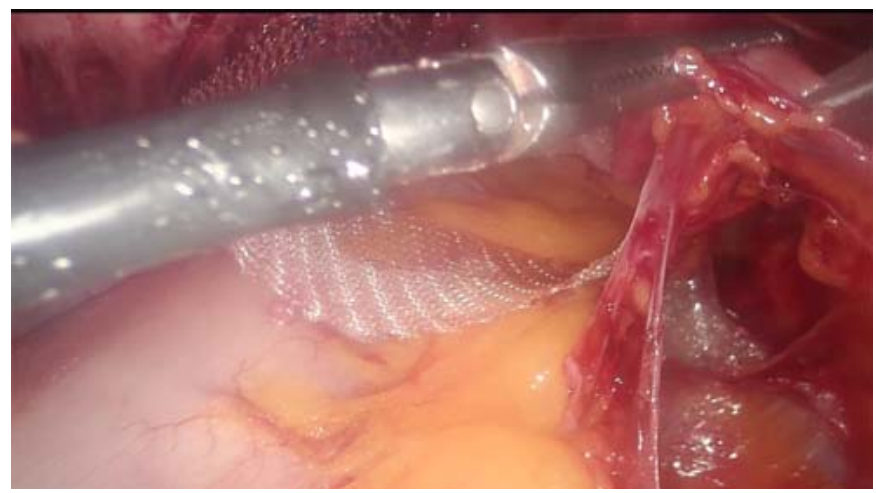

Figure 2: Tunnel under the peritoneum made by the LigaSure $^{\mathrm{TM}}$ Instrument

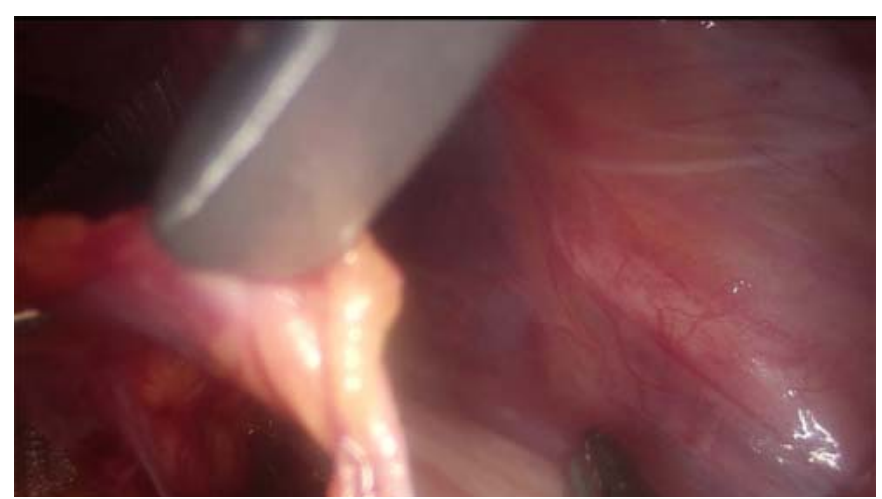

Figure 3: Checking the Right Ureteral Peristalsis

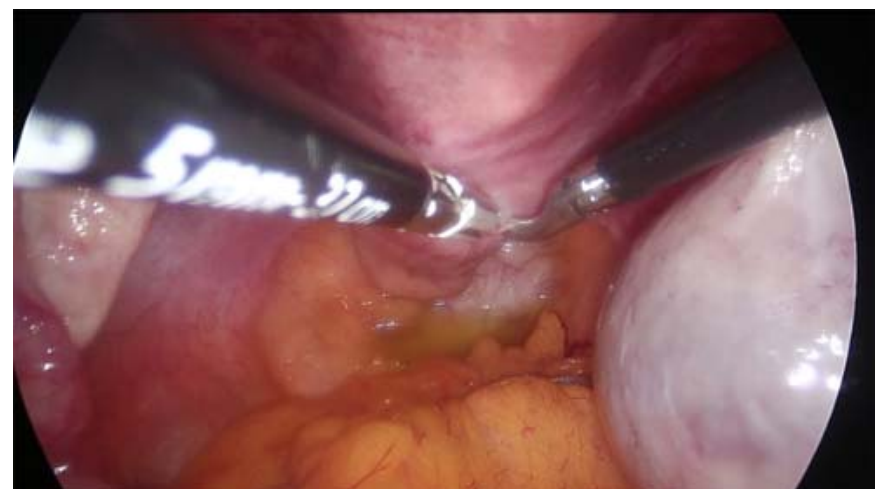

Figure 4: Preparing the posterior cervical wall 1.

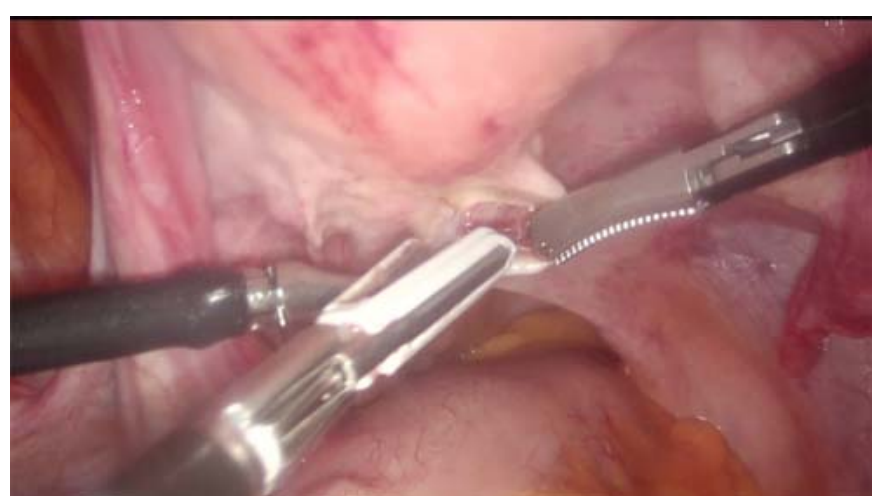

Figure 5: Preparing the posterior cervical wall 2.

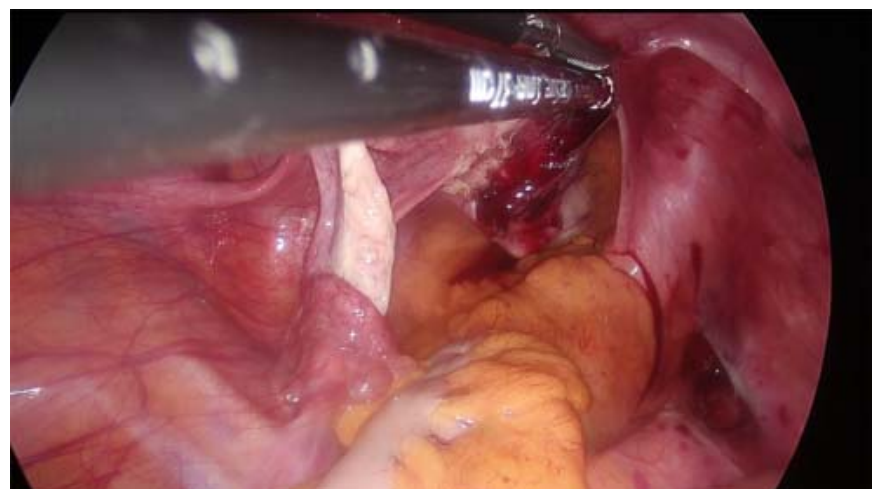

Figure 6: View of the posterior cervical wall after the preparation.

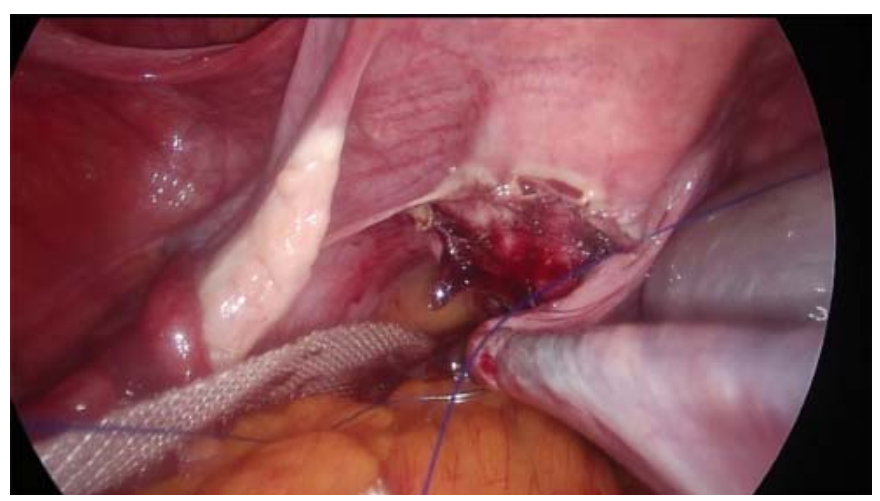

Figure 7: Passing the Mesh through the Tunnel

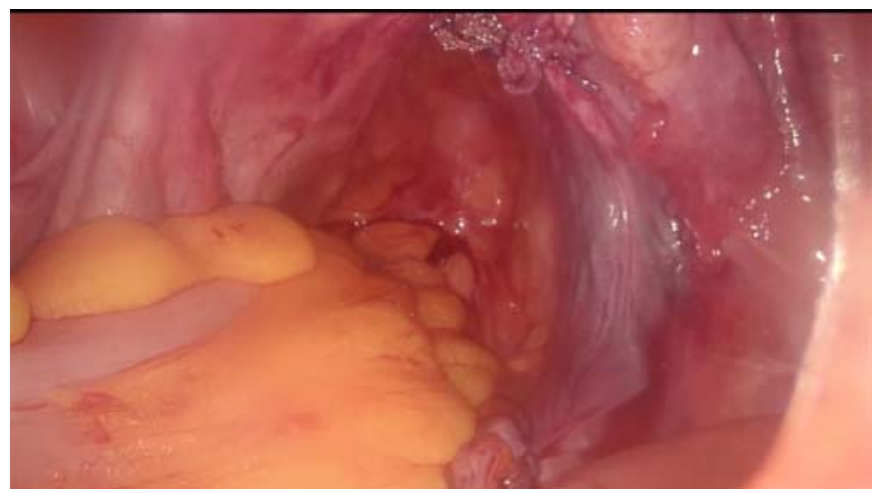

Figure 8: Final View 


\section{Results}

A total of 12 patients underwent LSTHS in our clinic between October 2017 and March 2019. All patient's complete data could be evaluated according to the study protocol. All of the patients had an apical prolapse of stage 2 or higher. Postoperative examination findings of these patients were obtained by examining the patients' files. The average follow-up period was between 6 and 21 months. Demographic and urogynecological history data are shown in table I.

The mean age of the patients who were included in the study was $51.25 \pm 18.11(35-84)$.

The mean operation was $147.5 \pm 43.97$ (90-240) minutes. None of our patients has had previous prolapses surgery. 9 of our patients have had vaginal deliveries. 2 of our patients have had cesarean sections. 1 of our patients was a virgin and there were not any pregnancies in her medical history.

2 of our patients have had prior operations due to umbilical hernia. The peritoneum was not ruptured in any surgery.

The average hematocrit decrease was $5.41 \pm 1.67 \%(2-8)$ but none of the patients needed any blood transfusion.

None of the patients developed incontinence during the postoperative period or sustained any bowel, bladder, vascular, or nerve injuries.

We have followed-up on some patients via telephone to inquire about their urinary habits and found out they have not observed any changes.

We performed patients' POP-Q measurements 6 months

Table I: Demographic characteristics and perioperative criteria of women who underwent LSTHS (no = 12)

\begin{tabular}{|c|c|c|}
\hline \multicolumn{2}{|l|}{ Age (year) } & $51.25 \pm 18.11(35-84)^{*}$ \\
\hline \multicolumn{2}{|l|}{ Parity } & $5.5 \pm 3.17(0-12)^{*}$ \\
\hline \multicolumn{2}{|c|}{ Average operation Time(min) } & $147.5 \pm 43.97(90-240)^{*}$ \\
\hline \multicolumn{2}{|c|}{ Blood loss (HTC, \%) } & $5.41 \pm 1.67(2-8)^{*}$ \\
\hline \multicolumn{2}{|c|}{ Length of stay in hospital(hours) } & $33.16 \pm 12.45(21-65)^{*}$ \\
\hline \multicolumn{2}{|c|}{ Concomitant surgery } & 4 Colporrhaphy anterior, 1 Myomectomy, 1 Cystectomy \\
\hline \multicolumn{2}{|c|}{ Body Mass İndex(kg/m2) } & $26.75 \pm 3.64(21-33)^{*}$ \\
\hline \multicolumn{2}{|c|}{ Menopause (n) (\%) } & $4(30)^{* *}$ \\
\hline \multicolumn{2}{|c|}{ Previous prolapse or continence surgery $(n)$} & none \\
\hline \multirow{2}{*}{ Mode of delivery: } & Vaginal & 9 \\
\hline & Cesarean section & 2 \\
\hline \multicolumn{2}{|l|}{ Smoking, $\mathrm{N}(\%)$} & $1(7)^{\star *}$ \\
\hline \multicolumn{2}{|l|}{ Hypertension } & 1 \\
\hline \multicolumn{2}{|l|}{ Hypothyroidism } & 2 \\
\hline \multicolumn{2}{|l|}{ Diabetes } & 0 \\
\hline \multicolumn{2}{|l|}{ Sexually active $(n)$} & 11 \\
\hline \multicolumn{2}{|c|}{ No sexual intercourse } & 1 \\
\hline \multicolumn{2}{|l|}{ Constipation (n) } & 0 \\
\hline \multicolumn{2}{|l|}{ Incontinence } & 0 \\
\hline \multicolumn{2}{|c|}{ Length follow-up (months) } & 6 \\
\hline \multicolumn{2}{|c|}{ Previous surgery history } & Umbilical hernia (2) \\
\hline \multicolumn{2}{|c|}{ Surgery complication } & none \\
\hline
\end{tabular}

Data were given as mean \pm standard deviation $(\min -\max ){ }^{*}$ or number (percentage) ${ }^{\text {** }}$

Table II: Objective anatomical outcomes of LSTHS at 6 months: ICS POPQ system (no = 12)

\begin{tabular}{|l|l|l|l|}
\hline ICS POP.Q measurement $\mathrm{cm}(\mathrm{n}=12)$ & \multicolumn{2}{l|}{$\mathrm{p}^{* *}$} \\
\hline POP-Q measurements & Preoperative $^{*}$ & Postoperative $^{*}$ & $\mathrm{p}=0.047, \mathrm{p}<0.05$ \\
\hline $\mathrm{Aa}$ & $1.5(-3,3)$ & $-2(-2,-1)$ & $\mathrm{p}=0.000, \mathrm{p}<0.001$ \\
$\mathrm{Ap}$ & $2(-3,3)$ & $-5(-7,-4)$ & $\mathrm{p}=0.028, \mathrm{p}<0.05$ \\
$\mathrm{Ba}$ & $2.5(-3,8)$ & $-1.5(-2,-1)$ & $\mathrm{p}=0.002, \mathrm{p}<0.01$ \\
$\mathrm{Bp}$ & $3.5(-2,8)$ & $-6(-8,-5)$ & $\mathrm{p}=0.003, \mathrm{p}<0.01$ \\
$\mathrm{C}$ & $2(-6,7)$ & $-6(-9,6)$ & \\
\hline
\end{tabular}

ICS POPQ: International incontinence society pelvic organ prolapse - Quantification system

*: Median (Min-Max). **: Wilcoxon Signed Ranks Test and Based on positive ranks. 
after surgery. A reduction was detected in the patients' degree of prolapse. As shown in table II, an objective anatomical improvement was observed (reduction in the ICS-POP-Q stage).

In addition, the mean values of each measurement point of the POP-Q showed significant improvement, especially point $\mathrm{C}$. The improvement in POP-Q points is presented in graphic 1. In addition to LSTHS, colporrhaphy anterior (CA) was performed in 4 patients, myomectomy was performed in 1 patient, and cystectomy was performed in 1 patient simultaneously.

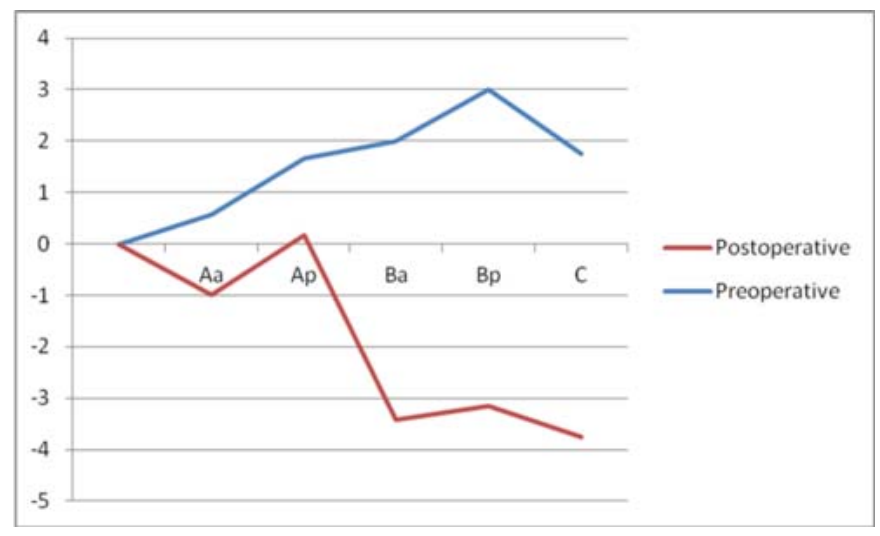

Graphic 1: $P O P-Q$ point change

When this study was done, all of the patients were called by phone and none of the patients had any complaints related to prolapse. There was no mesh erosion or recurrence of uterine prolapse throughout the 6-month postoperative follow-up period.

\section{Discussion}

In this study, it was found that the LSTHS procedure is both an efficacious and time-saving technique and does not result in any major adverse effects or unfavorable anatomic or functional outcomes.

As Baden and Walker pointed out, the restoration of the apex will improve the prolapse levels of other vaginal compartments (9). In our study, we found that restoration of uterine apex improves prolapse in all vaginal compartments.

There is no consensus on the level of vaginal dissection, the number of sutures, or the use of mesh.

Many authors stated that they do not prefer performing sacrocolpopexy because of its difficulties and long learning processes (10). In daily practice, members of the International Urogynecology Association (IUGA) and the American Urogynecologic Society (AUGS) differ in terms of vaginal level and the number of sutures (10). We reduced the number of sutures with this method. Therefore, we think that its implementation and the learning process will take a shorter time. Although some surgeons perform routine cystoscopy after the operations, we did not choose to. We preferred to show ureter dissection and peristalsis during surgery. We think it is sufficient to evaluate renal pelvic ectasia by USG in the post-op period.

Rectal and bladder injuries seem to increase with the depth of the vaginal dissection. It has also been reported that bowel and urinary dysfunction is less common (11). Less use of mesh and less tissue dissection may provide more results on this topic.

The findings we obtained in our study pointed out that the surgery is sufficient without extending prolene mesh to the upper posterior vaginal wall. As Antiphon et al. showed, the extension of the mesh to the posterior vaginal wall was unnecessary and could lead to complications. They also stated that it prolongs the operation time and increases the rate of complications such as dyspareunia (11). In our telephone conversation with our patients, we learned that none of our patients had dyspareunia complaints. We think the reason for this is that we did not extend the mesh to the upper vaginal wall.

In some studies, it was showed that after hysterectomy and pelvic reconstruction with a mesh, mesh erosion rates are as high as $20 \%$ (12). In our study, we did not perform a hysterectomy and any peritoneal incisions, and we did not observe any mesh erosions. Since the mean follow-up was 6 months, we do not know the long-term results.

As the previous studies clearly stated, it seems reasonable to avoid total hysterectomy, use a light-weight Type-I polypropylene mesh, delayed absorbable sutures or monofilament sutures and reduce the contact surface area between the vagina and the mesh. Based on this information, we performed accordingly.

The relationship between connective tissue diseases and prolapse has been discussed in the literature (13). Our two patients had a history of umbilical hernia. Patients with multiple hernias should also be evaluated for connective tissue disease.

One of our patients was a virgin and had no previous birth. But she had uterine myomatosis. Cases of uterine prolapse due to myomatosis have been reported in the literature (14). In nulliparous patients, uterine myomatosis should be evaluated in terms of prolapse etiology.

In literature, there are conflicting results in terms of urinary incontinence after prolapses surgery. However, our patients have not described any urinary incontinence after the operation. We think not dissecting the bladder may have had a role (15).

The retrospective design of this study and the lack of a control group were the limitations of this study. In addition, although our short term results are encouraging, the observation time was between 6 months and 2 years. The other limitation of this study is that we lack long term results. At the same time, more patients are needed to better evaluate the results. 


\section{Conclusion}

The present study found that LSC with minimal dissection was an efficacious and reconstructive approach, providing superior long-term anatomic and quality-of-life outcomes. Moreover, it was associated with shorter operative duration and no major intraoperative adverse events.

Well-designed randomized controlled trials should be carried out on larger populations to verify the results and provide evidence on the best technique for mesh attachment.

Conflict of interest "The authors declare that they have no conflicts of interest, commercial associations, or intent of financial gain regarding this research"

Acknowledgments: "None"

Funding: "None"

Author contributions: ES : Protocol/project development, data collection, management, data analysis, manuscript editing. EE: Data analysis, manuscript writing, translating.

\section{References}

1. Richardson AC, Lyon JB, Williams NL. A new look at pelvic relaxation. Am J Obstet Gynecol. 1976;126(5):56873. DOI: 10.1016/0002-9378(76)90751-1.

2. Brubaker L. Surgery for pelvic organ prolapse. In: Abrahams P, Cardozo L, Khoury S, Wein A, editors. Incontinence management. Paris, France: ICS Publications; 2005. p. $1371-402$

3. Marana HR, Andrade JM, Marana RR, Matheus de Sala M, Philbert PM, Rodrigues R. Vaginal hysterectomy for correcting genital prolapse. Long-term evaluation. J Reprod Med. 1999;44(6):529-34. PMID: 10394547.

4. Symmonds RE, Williams TJ, Lee RA, Webb MJ. Posthysterectomy enterocele and vaginal vault prolapse. Am J Obstet Gynecol. 1981;140(8):852-9. DOI: 10.1016/0002-9378(81)90074-0.

5. Marchionni M, Bracco GL, Checcucci V, Carabaneanu A, Coccia EM, Mecacci F, et al. True incidence of vaginal vault prolapse. Thirteen years of experience. J Reprod Med. 1999;44(8):679-84. PMID: 10483537.

6. Olsen AL, Smith VJ, Bergstrom JO, Colling JC, Clark
AL. Epidemiology of surgically managed pelvic organ prolapse and urinary incontinence. Obstet Gynecol. 1997; 89(4):501-6. DOI: 10.1016/S0029-7844(97)00058-6.

7. Coolen AWM, van Oudheusden AMJ, Mol BWJ, van Eijndhoven HWF, Roovers JWR, Bongers MY. Laparoscopic sacrocolpopexy compared with open abdominal sacrocolpopexy for vault prolapse repair: a randomised controlled trial. Int Urogynecol J. 2017; 28(10):1469-79. doi:10. 1007/s00192-017-3296-5.

8. Cosma S, Petruzzelli P, Chiadò Fiorio Tin M, Parisi S, Olearo E, Fassio F, Zizzo R, Danese S, Benedetto C. Simplified laparoscopic sacropexy avoiding deep vaginal dissection. Int J Gynaecol Obstet. 2018;143(2):239-45. DOI: 10.1002/ijgo.12632.

9. Baden WF, Walker T, editors. Surgical repair of vaginal defects. Philadelphia: JB Lippincott Company;1992.

10. O'Sullivan OE, Matthews CA, O'Reilly BA. Sacrocolpopexy: is there a consistent surgical technique? Int Urogynecol J. 2016;27(5):747-50. doi: 10.1007/ s00192-015-2880-9.

11. Antiphon P, Elard S, Benyoussef A. Laparoscopic Promontory Sacral Colpopexy: is the posterior, rectovaginal, mesh mandatory? Eur Urol. 2004;45(5):655-61. doi: $10.1016 /$ j.eururo.2004.01.004

12. Carramao S, Auge AP, Pacetta AM, Duarte E, Ayrosan P, Lemos NL, et al. A randomized comparison of two vaginal procedures for the treatment of uterine prolapse using polypropylene mesh: hysteropexy versus hysterectomy. Rev Col Bras Cir. 2009;36(1):65-72. doi: 10.1590/s010069912009000100012.

13. Callewaert B, Malfait F, Loeys B, De Paepe A. EhlersDanlos syndromes and Marfan syndrome. Best Pract Res Clin Rheumatol. 2008;22(1):165-89. doi: 10.1016/j.berh. 2007.12.005

14. Aniebue U, Nwankwo T. Complete utero vaginal prolapse in a woman with prolapsed submucous fibroid. Ann Med Health Sci Res. 2015;5(1):83-5. doi: 10.4103/2141-9248. 149795

15. El Hamamsy D, Fayyad AM. New onset stress urinary incontinence following laparoscopic sacrocolpopexy and its relation to anatomical outcomes. Int Urogynecol $\mathrm{J}$. 2015;26(7):1041-5. doi: 10.1007/ s00192-015-2641-9 